\title{
Characterization of integrated prophages within diverse species of clinical nontuberculous mycobacteria
}

\author{
Cody Glickman ${ }^{1,2^{*}} \mathbb{D}$, Sara M. Kammlade ${ }^{1}$, Nabeeh A. Hasan ${ }^{1}$, L. Elaine Epperson ${ }^{1}$, Rebecca M. Davidson ${ }^{1}$ and \\ Michael Strong ${ }^{1,2}$
}

\begin{abstract}
Background: Nontuberculous mycobacterial (NTM) infections are increasing in prevalence, with current estimates suggesting that over 100,000 people in the United States are affected each year. It is unclear how certain species of mycobacteria transition from environmental bacteria to clinical pathogens, or what genetic elements influence the differences in virulence among strains of the same species. A potential mechanism of genetic evolution and diversity within mycobacteria is the presence of integrated viruses called prophages in the host genome. Prophages may act as carriers of bacterial genes, with the potential of altering bacterial fitness through horizontal gene transfer. In this study, we quantify the frequency and composition of prophages within mycobacteria isolated from clinical samples and compare them against the composition of PhagesDB, an environmental mycobacteriophage database.
\end{abstract}

Methods: Prophages were predicted by agreement between two discovery tools, VirSorter and Phaster, and the frequencies of integrated prophages were compared by growth rate. Prophages were assigned to PhagesDB lettered clusters. Bacterial virulence gene frequency was calculated using a combination of the Virulence Factor Database (VFDB) and the Pathosystems Resource Integration Center virulence database (Patric-VF) within the gene annotation software Prokka. CRISPR elements were discovered using CRT. ARAGORN was used to quantify tRNAs.

Results: Rapidly growing mycobacteria (RGM) were more likely to contain prophage than slowly growing mycobacteria (SGM). CRISPR elements were not associated with prophage abundance in mycobacteria. The abundance of tRNAs was enriched in SGM compared to RGM.

We compared the abundance of bacterial virulence genes within prophage genomes from clinical isolates to mycobacteriophages from PhagesDB. Our data suggests that prophages from clinical mycobacteria are enriched for bacterial virulence genes relative to environmental mycobacteriophage from PhagesDB.

(Continued on next page)

\footnotetext{
* Correspondence: cody.glickman@cuanschutz.edu

${ }^{1}$ Center for Genes, Environment, and Health, National Jewish Health, Denver,

CO, USA

${ }^{2}$ Computational Bioscience Program, University of Colorado, Anschutz

Medical Campus, Aurora, CO, USA
}

C The Author(s). 2020 Open Access This article is licensed under a Creative Commons Attribution 4.0 International License, which permits use, sharing, adaptation, distribution and reproduction in any medium or format, as long as you give appropriate credit to the original author(s) and the source, provide a link to the Creative Commons licence, and indicate if changes were made. The images or other third party material in this article are included in the article's Creative Commons licence, unless indicated otherwise in a credit line to the material. If material is not included in the article's Creative Commons licence and your intended use is not permitted by statutory regulation or exceeds the permitted use, you will need to obtain permission directly from the copyright holder. To view a copy of this licence, visit http://creativecommons.org/licenses/by/4.0/ The Creative Commons Public Domain Dedication waiver (http://creativecommons.org/publicdomain/zero/1.0/) applies to the data made available in this article, unless otherwise stated in a credit line to the data. 


\begin{abstract}
(Continued from previous page)
Conclusion: Prophages are present in clinical NTM isolates. Prophages are more likely to be present in RGM

compared to SGM genomes. The mechanism and selective advantage of this enrichment by growth rate remain

unclear. In addition, the frequency of bacterial virulence genes in prophages from clinical NTM is enriched relative

to the PhagesDB environmental proxy. This suggests prophages may act as a reservoir of genetic elements bacteria

could use to thrive within a clinical environment.
\end{abstract}

Keywords: Mycobacteriophage, Nontuberculous mycobacteria, Prophage, Virulence, Growth rate

\section{Introduction}

Nontuberculous mycobacterial (NTM) infections can cause serious pulmonary disease that may become chronic and affect quality of life even leading to death [1]. Many of the mycobacterial species that cause NTM infections are ubiquitous in the environment and are known to thrive in built environments, including premise plumbing [2-4]. The mechanism by which these organisms, which have long been recognized as environmental, become clinical pathogens is an active area of research with prior studies exploring host susceptibility [5], geographic factors [6], and changes in genetic composition [7]. Mycobacterial species are categorized into two broad groups based on differing growth rates in culture; rapidly growing (RGM) and slowly growing (SGM) [8]. Of the six species/subspecies examined in this study $M$. avium, M. chimaera, and M. intracellulare are described as having a slow growth rate. M. abscessus subsp. massiliense, M. abscessus subsp. abscessus, and M. abscessus subsp. bolletii have a rapid growth rate [9].

Bacteriophages are viruses that infect bacteria and some are capable of transferring genetic material between bacteria through a process called transduction [10]. Mycobacteriophages are bacteriophages that target mycobacteria. Environmental bacteria are subject to external pressures to adapt their genomes through horizontal gene transfer, including bacteriophage transduction [10]. Temperate bacteriophages are capable of exhibiting both the lysogenic and lytic life cycles. The lysogenic cycle commonly involves a bacteriophage integrating genetic material into a bacterial genome and replicating in tandem until the integrated bacteriophage, also known as a prophage, transitions into a lytic life cycle. In addition to chromosomal integration, prophages can also exist within a host bacteria extrachromosomally [11]. During the lytic phase, the bacteriophage utilizes the bacterial cellular machinery to create new phage particles that are then released during bacterial cell lysis. Some of the newly created temperate phage particles package bacterial genes at a low frequency, which are subsequently transduced during a new infection [12]. This form of transduction is called specialized transduction, which is defined by the restriction of transducible bacterial genes to those flanking the integration site of the prophage. Another form of transduction, termed generalized transduction, occurs during the lytic phase when bacteriophages engaging in the headful packaging process include random pieces of the host bacterial DNA [13]. Both forms of transduction are thought to be rare events, however, given the immense number of bacteriophage-bacterial interactions, transduction events are estimated to occur at scale in the environment [14].

Virulence is a general term that describes a pathogen's invasive power, ability to overcome host defenses, and the replication efficiency of a pathogen within a host [15]. Prior studies have explored both the role of bacteriophages to increase virulence in bacteria and the effect of bacteriophage resistance on reduced virulence $[16,17]$. There is a selective advantage for bacteria that contain prophages with genetic elements capable of increasing propagative success and providing super-infection immunity [18]. It is possible that genes carried by mycobacteriophages during selective transduction events could impact virulence as seen in other bacteria such as Escherichia coli, Vibro cholerae, Corynebacterium diphtheriae, and Streptococcus pyogenes [19]. Prophages in these bacterial pathogens contain elements that contribute to quorum sensing, enzymatic functions, and extracellular toxicity $[19,20]$.

Here, we explore the frequency of integrated prophages in NTM genomes and characterize the composition of bacterial genes within predicted prophage elements. Genetic elements including tRNAs act as a potential insertion site for mycobacteriophages using a tyrosine-integrase [21]. Our hypothesis is that increased tRNA abundance is associated with an increased abundance of integrated prophages in mycobacteria due to there being more targets for integration with tyrosine-integrases. An important note is that other integrases can insert mycobacteriophages into genetic elements other than tRNAs and extrachromosomal prophages do not integrate at all [22]. CRIS PR elements provide a bacterial defense mechanism against viral challenge, and research in Cronobacter sakazakii suggests CRISPR elements with fewer spacers are more susceptible to prophage integration [23, 24].

To explore differences between clinical prophages and environmental mycobacteriophages, we utilized PhagesDB, which is a data repository mostly composed of mycobacteriophages isolated from the environment [25]. 
A majority of these mycobacteriophages were identified using a phage plaque screening assay to identify lytic and temperate mycobacteriophages capable of infecting the non-pathogenic species $M$. smegmatis. Mycobacteriophages from PhagesDB are organized into sequence clusters, indicated by letters (A-Z), based first on sequence similarity and then shared functional protein families [26]. Lettered clusters typically exhibit similar lifestyle and functional behavior. Prior research has suggested PhagesDB mycobacteriophages clusters K, G, and A are capable of infecting clinical NTM including RGM and SGM [27, 28]. Our hypothesis is that prophages from genomes of clinical isolates may be enriched for bacterial virulence genes compared to environmental mycobacteriophages from PhagesDB. Exploring this hypothesis helps us to investigate the ability of prophages to act as a genetic repository for bacterial virulence genes within clinical NTM genomes.

\section{Methods}

\section{Bacterial genome assembly and isolate datasets}

In this study, we utilized two publically available datasets. First, we downloaded the complete genomes from two NTM species in the NCBI assembly database (Supplementary File 1), M. abscessus and M. avium ( $n=53$ complete genomes). These species were selected because they represent the two most common NTM species of clinical significance [29]. All complete genomes were isolated from clinical sources, with the exception of an $M$. avium isolate from a hospital water source [30]. Our decision to look at complete genomes was driven by the desire to examine bacteriophage trends between species regardless of assembly status (complete vs. draft genome).

The second dataset includes a collection of 318 NTM isolates from 168 individuals diagnosed with cystic fibrosis (CF) [31]. All samples were cultured on solid media, converted to glycerol stock aliquots, and the DNA was extracted for whole genome sequencing using an optimized mycobacterial DNA preparation protocol $[31,32]$. Bacterial isolates were collected in a longitudinal manner, however, only one isolate genome per patient per species was retained for this prophage analysis. Fourteen patients had multiple NTM species, and in these cases, one isolate from each species was retained ( $n=182 \mathrm{draft}$ genomes). This dataset includes six different mycobacterial species and subspecies: $M$. abscessus subsp. massiliense, M. abscessus subsp. abscessus, $M$. abscessus subsp. bolletii, M. avium, M. chimaera, and M. intracellulare (Table 1).

Paired-end reads from draft genomes in the CF dataset were assembled using Unicycler into contiguous sequences (contigs), known as draft genomes, with a median N50 ranging from $\sim 82$ kilobases to $\sim 216$ kilobases (Table 1) [33]. Numbers of contigs in the draft genome assemblies ranged from 37 to 336. Analysis of assembly completeness based on N50 length and the number of contigs metrics revealed three outliers, which were removed from downstream analysis. To understand if assembly fragmentation affected prophage prediction in our draft genomes, we explored the edge cases, which are defined as predicted prophages within 100 bases of either end of a contig. Given the distinctions in assembly statistics by growth rate, linear mixed modeling was performed to calculate statistical significance of assembly features against prophage frequency.

Species identifications of draft genomes were determined using a method of average nucleotide identity (ANI) as described previously [31] and sequence reads mapped to active reference genomes to generate phylogenies [34, 35]. Reads were mapped using Bowtie2 software [36] and single nucleotide polymorphisms (SNPs) were determined using Samtools mpileup [37]. Mycobacterial genotypes were concatenated and used to make phylogenetic trees using maximum likelihood with 1000 bootstrap replicates in Randomized Axelerated Maximum Likelihood-Next Generation (RAxML) [38]. SNP distances between groups in the tree file were used to perform PERMANOVA analysis in R.

Table 1 Summary statistics of prophages predicted in the NTM draft genomes assemblies. Median counts of tRNA, N50 Lengths, and counts of contigs in NTM draft genomes are shown with the ranges in parenthesis. The edge case average is the total number of edge cases divided by the draft genome count

\begin{tabular}{|c|c|c|c|c|c|c|c|}
\hline Species & $\begin{array}{l}\text { Genomes } \\
\text { Count }\end{array}$ & $\begin{array}{l}\text { Genomes } \\
\text { with Phage }\end{array}$ & $\begin{array}{l}\text { Predicted } \\
\text { Prophages }\end{array}$ & $\begin{array}{l}\text { Median tRNA } \\
\text { Count }\end{array}$ & Median N50 Length & $\begin{array}{l}\text { Median } \\
\text { Contig } \\
\text { Count }\end{array}$ & $\begin{array}{l}\text { Edge Cases } \\
\text { Average }\end{array}$ \\
\hline M. avium & 43 & 1 & 1 & $57(54-108)$ & $81,670(56262-116,826)$ & $206(126-336)$ & 1.07 \\
\hline M. chimera & 11 & 1 & 1 & $55(49-81)$ & $86,644(78123-101,816)$ & $193(116-251)$ & 1.00 \\
\hline M. intracellulare & 23 & 3 & 4 & $52(50-54)$ & $101,938(82877-131,830)$ & $104(83-145)$ & 0.13 \\
\hline M. abscessus & 76 & 42 & 51 & $48(45-116)$ & $168,941(87141-327,102)$ & $67(41-191)$ & 2.60 \\
\hline M. bolletti & 4 & 3 & 3 & $48.5(48-49)$ & $208,710(140476-226,296)$ & $45(37-59)$ & 2.50 \\
\hline M. massiliense & 25 & 11 & 12 & $51(46-84)$ & $215,865(97542-413,240)$ & $53(39-143)$ & 2.84 \\
\hline
\end{tabular}


Phylogenetic trees were annotated and visualized with ggtree [39]. Additional tree file manipulations and visualizations were performed with ETE3 [40].

ARAGORN v1.2.38 was used to quantify the tRNAs in the mycobacterium [41]. CRISPR identification was performed using CRT with default parameters (minimum 3 repeats, minimum length 19 base pairs, maximum length 38 base pairs) [42].

\section{Integrated prophage discovery}

Prophage discovery was performed using the agreement of two prophage discovery tools, Phaster and VirSorter $[43,44]$. Phaster and VirSorter use different sequence similarity methods against known viruses to find prophage elements within bacterial genomes. Phaster was utilized because of the ability to consider the completeness of a putative prophage region through identification of elements such as attachment sites. VirSorter differs from Phaster in that it does not find attachment sites, however VirSorter outperforms other tools on prophage identification in fragmented genomic datasets [44]. A custom application programming interface (API) script was used to identify prophages from the Phaster web server, whereas VirSorter prophage identification was performed locally. The output of Phaster contains three confidence levels for a prophage prediction with "intact" possessing the strongest support, "questionable" having some support, and "incomplete" being the least confident. VirSorter also ranks prophage predictions into three numeric levels (4 strongest, 5 middle, and 6 low support) in addition to predicting individual contigs from the draft genomes as stand-alone viruses (1 strongest, 2 middle, and 3 low support). Confidence levels of Phaster predictions were manually set to the scale of VirSorter prophages. Only Phaster predicted prophages with overlapping ranges between the two tools were retained. Predicted prophages were further filtered by requiring identified attachment sites from Phaster, at least 10 proteins from the predicted prophage match against the PhagesDB protein database, and the presence of an integrase gene [45]. Edge case identifications incorporate all predicted prophages, not only those selected by both prophage prediction tools.

Dereplication of predicted prophage elements was performed using VSEARCH [46] to identify genetically identical prophages between different genomes, which may be evidence of transmission or contamination.

\section{Prophage identification and genome annotation}

PhagesDB uses MMSEQ2 to cluster mycobacteriophage gene products into gene "phamilies", then uses Splitstree to create functional clusters based on presence or absence of gene "phamilies" [47]. The version of PhagesDB used was filtered to only contain mycobacteriophages.
Our approach to assign predicted prophages to clusters began by calculating average nucleotide identity (ANI) using the MUMmer toolset against known mycobacteriophages from PhagesDB [48]. Per prior work, an ANI greater than $60 \%$ and with a genomic coverage of at least $50 \%$ would cluster a phage [49]. Following ANI clustering, we selected BLASTp because the gene "phamilies" are not included in the data API, and the parameters of MMSEQ2 cluster are not clearly defined in prior works [26]. A match was based on DIAMOND Blastp homology with default parameters (e-value $<1 \mathrm{e}-12$, query coverage $>=70$, identity cut-off $>=70$ ) against proteins from PhagesDB downloaded using the PhagesDB API [50]. A minimum of 5 protein matches from the predicted prophage to a cluster are required for cluster assignment, otherwise all unidentified prophages are assigned to no cluster. Gene products of predicted prophages were identified using Prodigal [51]. The aggregation of cluster identifications from gene hits was used to determine the projected cluster of the predicted prophage. The clustering of predicted prophages was visualized using RAWGraphs.io [52].

Bacterial genes within predicted prophages were annotated using Prokka [53] with an additional virulence gene and phage protein database combining VFDB, Patric-VF, and PhagesDB proteins added as a parameter [25, 53-55]. Proteins were predicted using Prodigal and subsequently annotated with a combination of DIAMOND BLASTp and HMMscan using default parameters [50, 51, 56]. Prodigal has a default minimum peptide length of 90 [51]. Pairwise significance testing, comparing the abundance of virulence genes in the PhagesDB and the predicted prophages, was performed using a $\mathrm{z}$-score test for two population proportions with binary success defined as having any bacterial virulence gene in the genome.

Prophage counts by mycobacterial species were visualized using pandas (version 0.20.3) and matplotlib (version 2.1.0) $[57,58]$. Pairwise significance testing comparing the abundance of prophages between species growth rate was performed using a z-score test for two population proportions with binary success defined as having a prophage with more than 10 gene matches against PhagesDB in the genome.

Pangenome analyses were performed using Roary [59] to identify core genes (present in $>95 \%$ of prophages) and shell genes (present in between 15 and 95\% of prophages) amongst all ORFs in the predicted prophages $(n=96)$. In addition, assigned PhagesDB clusters of predicted prophages with more than five prophages per cluster were subjected to another pangenome analysis $(n=81)$.

\section{Results}

\section{Abundance of prophages in NTM species}

Prophages were predicted in 81 of the 235 clinical NTM genomes $(37.7 \%$ of complete genomes and $33.5 \%$ of draft 
genomes). Within the 81 genomes with prophages, a total of 96 unique prophages were identified. The interquartile range of prophage lengths across all species was $\sim 38$ kilobases to $\sim 58$ kilobases. A Kruskal-Wallis test of prophage lengths by growth rate failed to reject the null hypothesis suggesting no difference in the size of integrated prophages by growth rate $(\mathrm{H}=1.93, p=0.165)$. We predicted integrated prophage elements in both RGM and SGM, however predicted prophages more likely to be found within RGM than in SGM ( $\mathrm{F}=6.71$, $p=1.96 \mathrm{e}-11 \mathrm{draft}$ genomes and $\mathrm{F}=3.83, p=1.29 \mathrm{e}-04$ complete genomes, proportions z-test, Fig. 1). In the RGM M. abscessus subsp. abscessus, 61 out of 109 (56.0\%) clinical NTM have predicted prophage elements, while of the SGM, only 2 out of $63 \mathrm{M}$. avium (3.2\%), 3 out of $23 \mathrm{M}$. intracellulare (13\%) and 1 out of $11 \mathrm{M}$. chimaera (9.1\%) draft genomes have intact prophage elements. The relative genomic locations of the predicted prophages within the complete genome dataset are shown in Supplementary Figure 1.

To test if the prophage discovery tools are biasing prophage predictions in longer contiguous sequences (i.e. complete genomes), we quantified the number of edge cases by species in the $182 \mathrm{draft}$ genomes. The number of edge cases are normalized by genome count per species to generate an average number of edge cases per species (Table 1). Edge cases are only quantified in the draft genome assemblies because the complete genomes are assumed to have only one contiguous sequence (Table 2). Linear mixed models of prophage frequency by number of contigs $(\mathrm{Z}=-3.27, p=1.1 \mathrm{e}-03)$, N50 Length $(Z=1.94, p=0.052)$, and number of contigs $>1500$ base pairs $(\mathrm{Z}=-4.10, p=4.19 \mathrm{e}-04)$ approached or achieved significance. However, additional post-hoc testing of significant linear mixed models revealed no significant correlations suggesting assembly fragmentation likely had little effect on the number of predicted prophages between species.

Thus, in future figures, draft and complete genomes are combined for downstream analysis.

\section{Predicted prophages annotated using existing mycobacteriophage clusters}

PhagesDB is a database containing mycobacteriophage genomes categorized into clusters and sub clusters based on sequence similarity and shared functional genes. Predicted prophages in our study were annotated across 15 clusters (Fig. 2) using ANI and protein sequence similarity. The RGM had prophages in all 15 lettered groups, and SGM had 3 lettered groups. Most prophages (31.3\%) fell into the "no cluster" category, which represents prophages matching other prophages without a defined cluster or not being assigned a cluster using ANI or BLASTp. The number of errors associated with the ANI assignment to lytic clusters is on average nearly double that of prophages assigned to lysogenic clusters $(22,171$

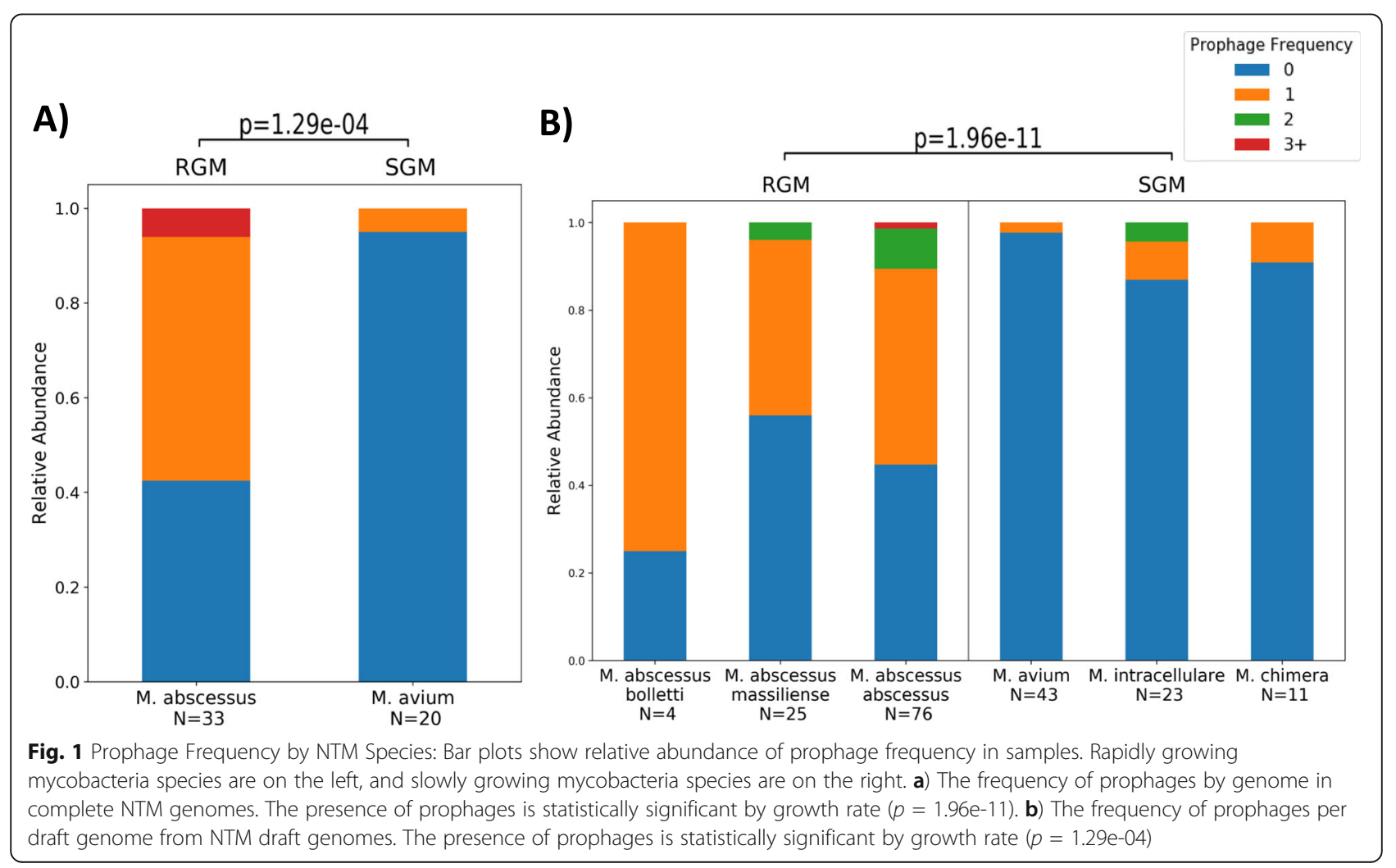


Table 2 Summary statistics of predicted prophages within complete NTM genomes selected from the NCBI assembly database. Complete genomes are not fragmented into contigs, thus N50 statistics, contig count, and edge cases are not applicable. Additional information about these genomes are available in Supplementary File 1

\begin{tabular}{lllll}
\hline Species & Genomes Count & Genomes with Phage & Predicted Prophages & Median tRNA Count \\
\hline M. avium & 20 & 1 & 1 & $59(54-59)$ \\
M. abscessus & 33 & 19 & 23 & $49(46-104)$ \\
\hline
\end{tabular}

lytic against 12,385 lysogenic base pair errors, $p=0.098$, Mann Whitney U).

Across the entire dataset (draft and complete genomes), the 96 identified prophages consisted of 84 genotypically unique sequences. Predicted prophages that clustered with another predicted prophage were from the same species, which could represent a shared evolutionary sequence or a common insertion site.

The pangenome analysis using all predicted prophages resulted in no core genes shared among 96 unique prophages. The largest number of prophages that shared a gene was 25 prophages and the gene function was undefined. Supplementary Table 1 details the pangenome analyses, including shell gene counts between prophages discretized by assigned PhagesDB cluster. No core genes are found in any lettered cluster pangenome analysis. Hypothetical proteins of unknown function are most commonly shared shell genes within the predicted prophages.

\section{Prophage annotation results and virulence genes by species}

There was an average of 68 open reading frames (ORFs) predicted within each of the 96 prophages from the draft and complete genomes. The average peptide length of ORFs was 216 (24-1937) amino acids. The total number of ORFs among all prophages was 6550. Of these predicted ORFs, $65.24 \%$ were labeled hypothetical proteins without a known function. Almost half of the ORFs (48.89\%) were annotated using sequence similarity to a protein within the PhagesDB mycobacteriophage database. ARAGORN identified 156 tRNAs with tRNAthreonine as the most abundant, comprising $16.0 \%$ of the total. The number of ORFs annotated as bacterial genes in the dataset, not including tRNAs, was 245 (3.74\%). Of ORFs identified as bacterial genes, 66 ORFs (1.01\% of all ORFs) were predicted to derive from bacterial virulence genes.

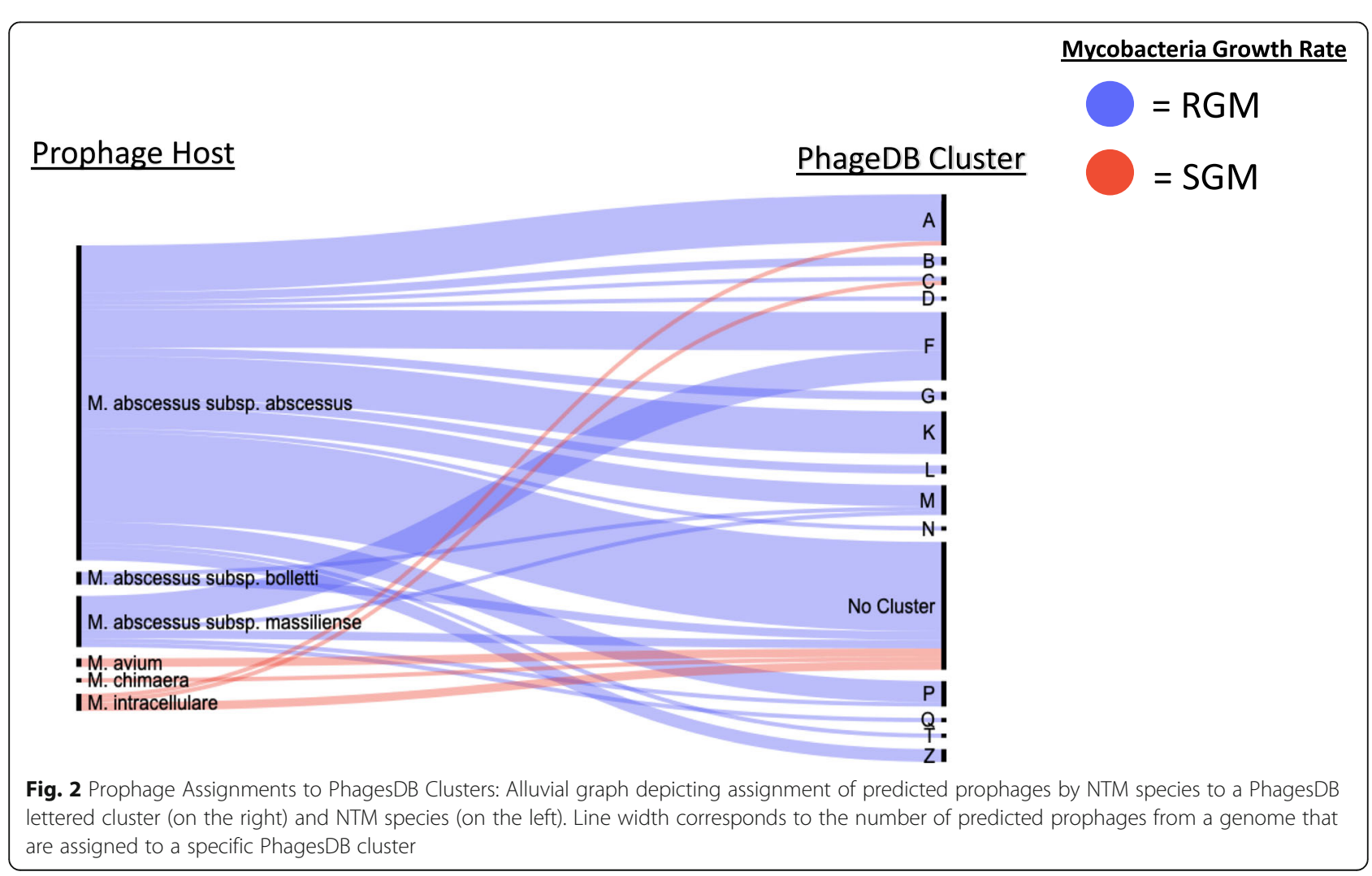


Virulence gene annotations within the predicted prophages comprised about 1\% of the ORFs. The 66 predicted ORFs annotated as bacterial virulence genes were present across 39 prophages from 37 different NTM genomes. Figure 3 highlights the presence/absence of bacterial virulence gene within the predicted prophages across mycobacterial species. Of the genes annotated as virulence genes by VFDB or Patric-VF, 51.5\% were originally identified in Mycobacterium tuberculosis and $16.7 \%$ derive from Salmonella enterica (Supplementary File 2).

PhagesDB contains 1795 mycobacteriophages. Within these mycobacteriophages, 187 mycobacteriophages contained 249 virulence genes or $0.13 \%$ of all predicted ORFs. For a fairer comparison of virulence gene abundance between our predicted prophages and PhagesDB, lysogenic mycobacteriophages were subset from PhagesDB leaving 1271 mycobacteriophages and 122,405 ORFs. Within these 1271 mycobacteriophages, 158 mycobacteriophages contain 220 annotated virulence genes $(0.18 \%$ of all ORFs). We found that bacterial virulence genes are more likely to be present within prophage elements derived from clinical sources than from all mycobacteriophage genomes in PhagesDB isolated from the environmental M. smegmatis and the subset selected from lysogenic mycobacteriophages $(\mathrm{F}=8.89$, $p=6.16 \mathrm{e}-19$ and $\mathrm{F}=6.73, p=1.73 \mathrm{e}-11$, proportions $\mathrm{z}$ - test, Fig. 4). The relative locations and functional annotations of bacterial virulence genes in the predicted prophages, as well as PhagesDB mycobacteriophages are shown in Fig. 5. The location of the virulence genes in the predicted prophages, with $43.9 \%$ within $10 \%$ of either end of the predicted prophage, suggests either specialized transduction, where genes flanking the prophage insertion site are transduced or an error in the predicted prophage range (Fig. 5). 125 bacterial virulence genes (50.2\%) are within $10 \%$ of mycobacteriophage ends in the PhagesDB database (Fig. 5). The presence of virulence genes in clinical isolates is significantly enriched even when these virulence genes near the ends are removed from only the predicted prophages $(\mathrm{F}=5.03, p=4.85 \mathrm{e}-7$ and $\mathrm{F}=3.37$, $p=7.44 \mathrm{e}-4$, proportions z-test).

\section{Mycobacterium phylogeny influences on prophage frequency}

To test if NTM draft genomes with prophages are more evolutionarily similar to each other than to NTM draft genomes without prophage, we performed a PERMANOVA test of phylogenetic distance metrics. The genome wide genetic distances of $M$. abscessus subsp. abscessus draft genomes are more similar within the groups: with and without prophages, than between groups $(\mathrm{F}=2.17, p=$ 0.026, PERMANOVA). Supplementary Figure 2 displays

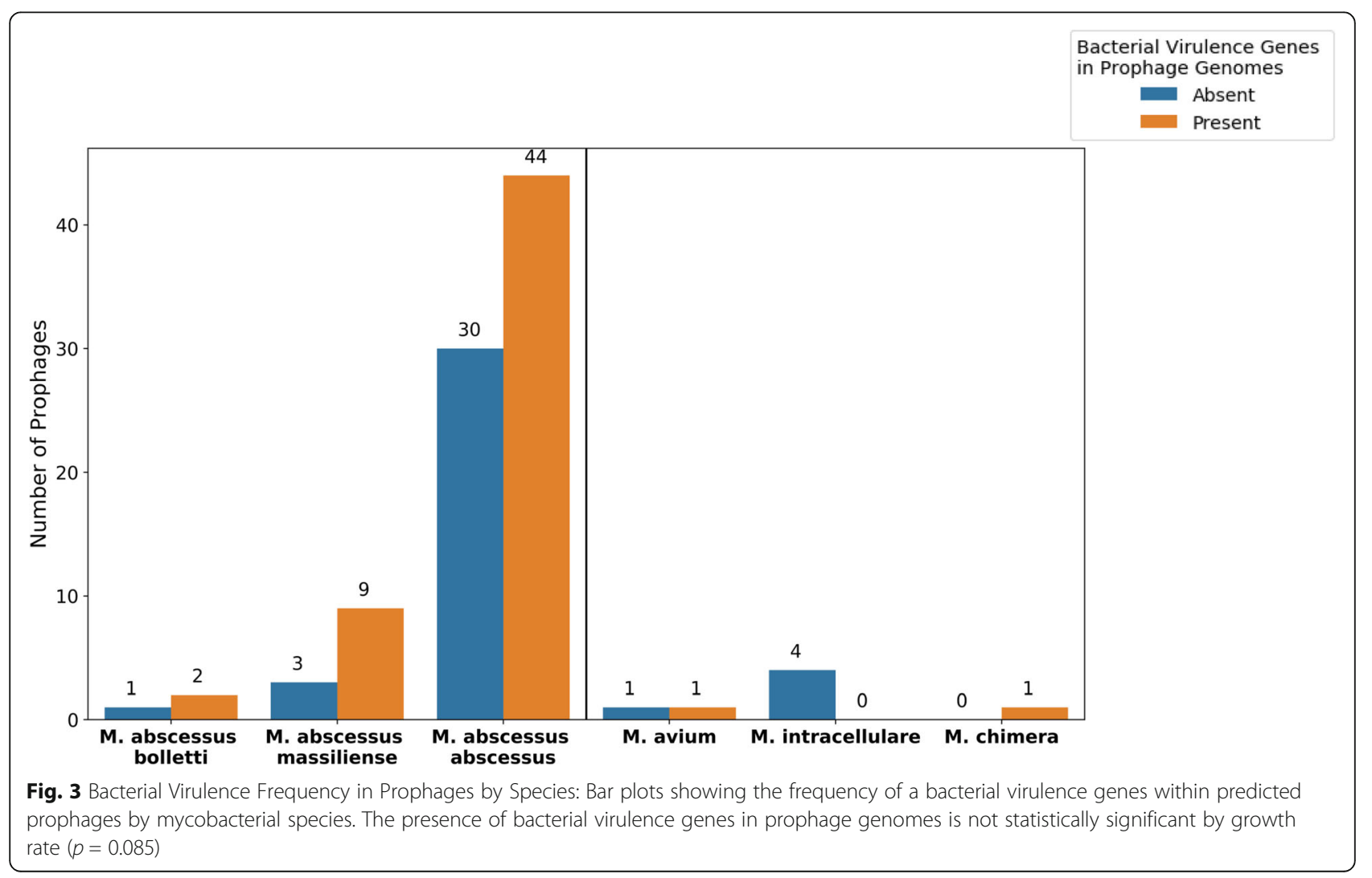




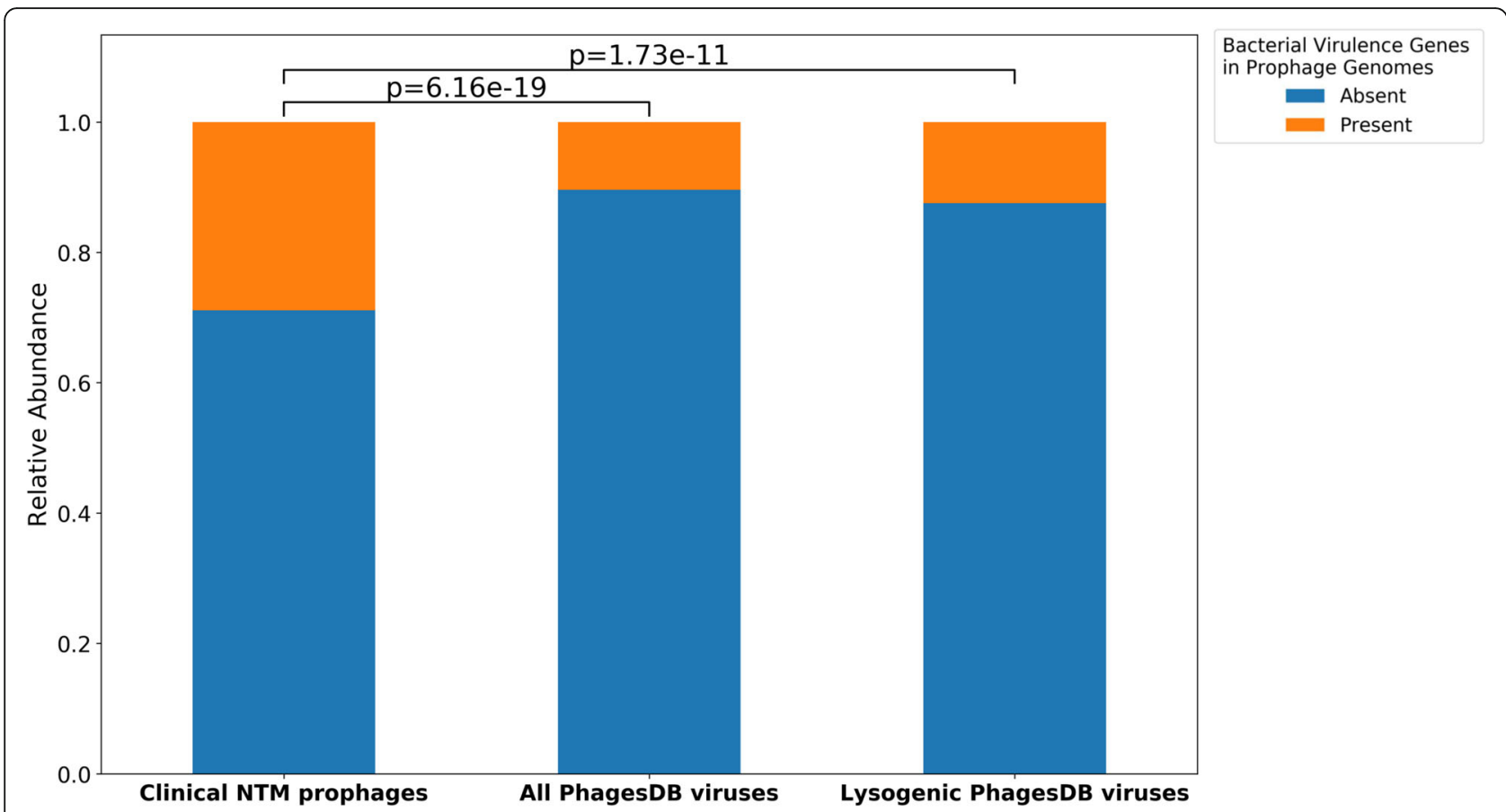

Fig. 4 Bacterial Virulence Frequency in Prophages by Data Source: Bar plots showing relative abundance of bacterial virulence genes within viral genomes from our predicted prophages, mycobacteriophages from PhagesDB, and lysogenic mycobacteriophages of PhagesDB. The presence of bacterial virulence genes in the genomes of our predicted prophages is statistically significant against the presence of bacterial virulence genes in both the full PhagesDB mycobacteriophages and the lysogenic PhagesDB mycobacteriophages ( $F=8.89, p=6.16 \mathrm{e}-19$ and $\mathrm{F}=6.73, p=1.73 \mathrm{e}-$ 11, proportions z-test)

the distribution of prophages in M. abscessus subsp. abscessus and M. abscessus subsp. bolletti in the context of the bacterial phylogeny. The distributions of predicted prophages in other species are shown in Supplementary Figures 3 and 4.

CRISPR elements have the capability to protect a bacterium against prophage integration. CRISPR elements were present in only 4 of the $53(7.5 \%)$ complete genomes and 12 of the 182 (6.6\%) draft genomes. Presence of CRISPR elements was not associated with the number of prophages $(\mathrm{H}=0.0092, p=0.92$, Kruskal-Wallis $)$. The abundance of tRNAs in the mycobacterial genomes was significantly different by growth rate with slowly growing mycobacterial species having a greater number of tRNAs $(\mathrm{H}=89.43, p=3.18 \mathrm{e}-21$, Kruskal-Wallis). In addition, the relationship of tRNAs and prophage frequency corrected by species reveals a positive linear correlation only in $M$. abscessus $\left(\mathrm{R}^{2}=0.34, p=3.21 \mathrm{e}-4\right)$.

\section{Discussion}

In this study, we detail the frequency of prophages within six different species of NTM from 182 draft genomes and 53 complete genomes. Prophages in this study are predicted using an ensemble approach combining two tools that identify prophages in different ways [60]. Prophage prediction in this study does not guarantee viruses capable of excising, but is indicative of integrated elements that contribute to the evolution of the host.

The number of prophages found in this study within mycobacteria with a rapid growth rate is greater relative to the number found in slowly growing mycobacteria (Fig. 1). Assemblies of SGM are more GC rich than RGM, which is correlated with higher contig numbers (Table 1). Prophage identification does not appear to be driven by assembly fragmentation as evident by the edge case ratio. A higher edge case ratio in RGM compared to SGM means that if prophages are missing from the assembly they are more likely to be missing in RGM. In addition, the correlation between the number of contigs, median contig length, number of sequences $>1500$ base pairs, and the number of predicted prophages did not hold during post-hoc testing. This further supports the notion that assembly fragmentation is not affecting the identification of prophages.

Our analyses revealed that the presence of CRISPR elements are rare in NTM, as only 16 of 235 samples (6.8\%) contained CRISPR elements. Prior studies of CRISPR elements in mycobacteria found loci with greater than 5 repeats only in M. tuberculosis, M.bovis, 


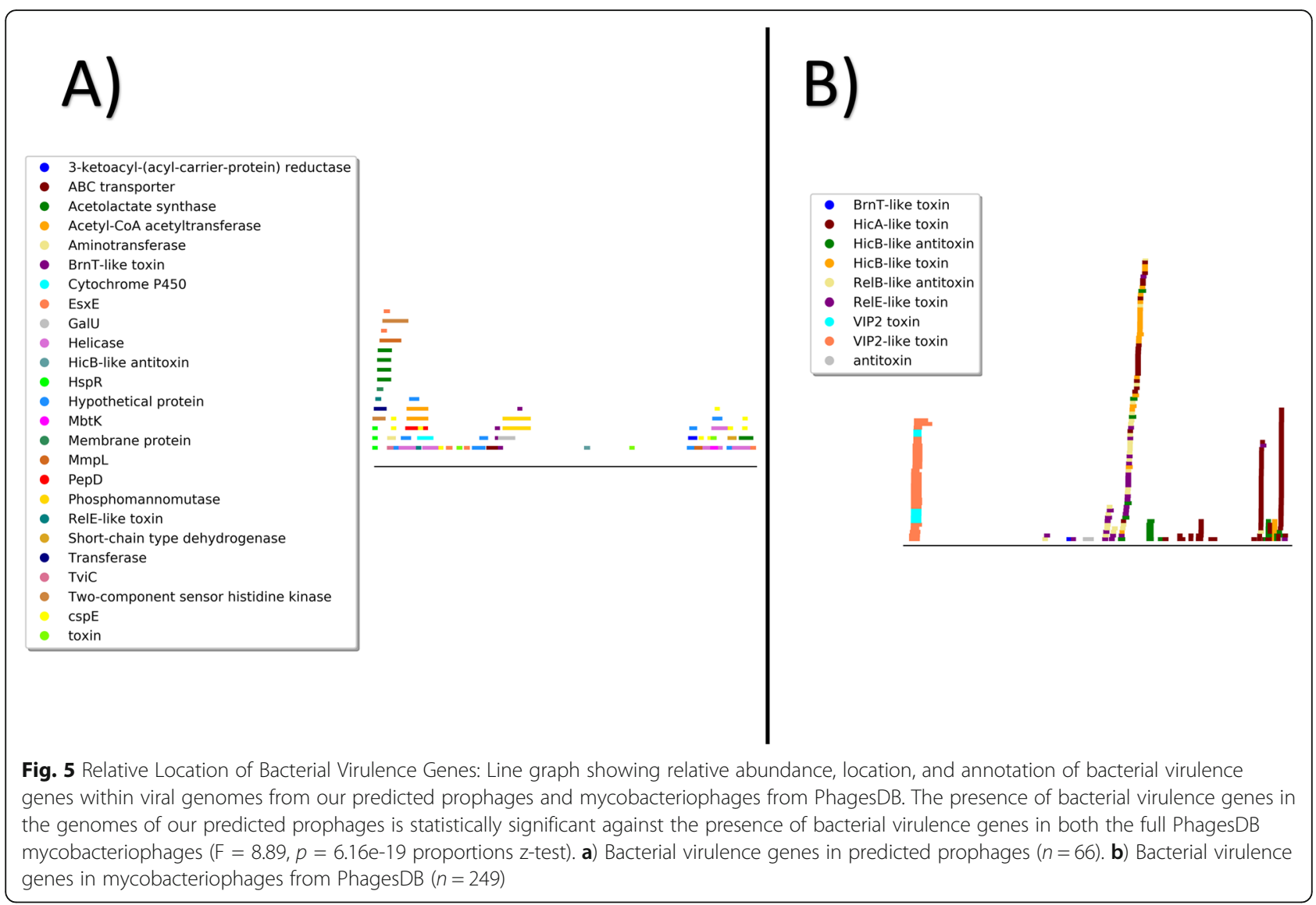

and $M$. avium species [61]. Prophage frequency did not correlate with the presence of CRISPR elements, although the sample size of mycobacteria with CRISPR elements could be a limiting factor within this study.

tRNAs can act as an insertion site target for prophages using a tyrosine-integrase [21]. Our hypothesis was that an increased abundance of tRNA would result in more prophages due to the increase in potential target integration sites for tyrosine-integrases in mycobacteriophages. The abundance of tRNAs in SGM is enriched relative to RGM. This is counterintuitive to our hypothesis considering the frequency of prophages by growth rate. We did observe a positive correlation of tRNA counts and prophages within $M$. abscessus subsp. abscessus genomes though our sample size is limited for draft genomes with higher numbers of prophages. Interestingly, the increased abundance of tRNAs in SGM did not translate to increased frequency of prophages compared to RGM, which may be a result of prophages using a different integrase or SGM inhibiting the integration of prophages using another mechanism [22].

The evolutionary advantage for RGM to have more prophages is unclear in the context of this study. Prior studies in E. coli have shown an increased growth rate linearly corresponds to an increase in burst size of phages from the host [62]. Additionally, the increased abundance of host bacteria may achieve a threshold necessary for bacteriophage replication thus allowing the continuation of integrated prophages in subsequent colonies of RGM [63]. M. smegmatis, the nonpathogenic model organism that is commonly used to isolate mycobacteriophages is a RGM [27].

Many predicted prophages did not share significant similarity to other known mycobacteriophages. The low similarity between the predicted prophages and the known mycobacteriophages could be a result of genetic mosaicism or genetic degradation of the integrated element. Of those that were assigned to a cluster, 12 prophages were assigned to clusters by ANI that typically exhibit a lytic life cycle. Changes to the threshold of amount errors allowed in cluster assignment may affect the clustering of predicted prophages. The prophages predicted in this study do not share a core genome reflecting the wide variability of viruses. The gene shared amongst the most prophages was present in only 25 prophages. This gene and many others that are highly shared have no defined function and are labeled hypothetical proteins. Of note, the high mosaicism found in 
this study supports prior explorations of mycobacteriophage genomes. Pedulla et al. annotated 10 novel mycobacteriophages and noted the abundance of previously defined bacterial genes within viral genomes $(3.1 \%$ of ORFs) including those homologs with the potential to elicit an immune response in humans against $M$. tuberculosis and M. leprae [64].

Annotations of the predicted prophage elements supports the rarity of transduction events, with $3.74 \%$ of ORFs predicted annotated as bacterial genes. Also, virulence genes were more abundant within prophages from clinical NTM genomes than environmental mycobacteriophages cataloged in PhagesDB (1.01\% clinical NTM vs $0.13 \%$ PhagesDB). Our results support our hypothesis suggesting prophages could act as a reservoir of bacterial genes important for virulence (Fig. 4). The location of the virulence genes near the ends of predicted prophages suggest either specialized transduction, where genes flanking the prophage insertion site are transduced or an error in the predicted prophage range (Fig. 5). The percentage of virulence genes identified near the ends of prophages and mycobacteriophages is similar (43.9\% in prophages and $50.2 \%$ in mycobacteriophages) and removing the virulence genes on the ends from the predicted prophages maintained the observation that bacterial virulence genes are more likely to be present within prophage elements derived from clinical sources than from mycobacteriophages of PhagesDB.

Though clinical NTM are known to display different levels of virulence, even within a species, it is unclear if virulence genes within prophage elements affect patient outcomes [65]. Presence of virulence genes alone does not mean these genes are actively expressed, and the presence of a prophage in a genome does not guarantee a functional or excisable virus. Further studies of RNA transcription of mycobacteria with prophages would be helpful in characterizing the expression of phage genes. In addition, our study relied on PhagesDB as an environmental proxy of mycobacteriophage. Future studies exploring prophage frequency within environmental isolates of mycobacteria are needed to directly compare prophage susceptibility of clinical and environmental NTM genomes.

This study demonstrates the presence of prophages in clinical species of mycobacteria. Prophages offer a mechanism for the genetic mosaicism of mycobacteria which have been observed to lack a distributed conjugal transfer (DCT) protein [66]. An increase in the genetic fluidity of a bacterial infection by prophage elements can impact patient outcomes as seen in other pathogens [67]. Mycobacteriophages may contribute to the pathogenic potential of environmental mycobacteria by acting as an external genetic reservoir. Additional work is needed to understand the role of mycobacteriophages in shaping the dynamics of mycobacterial infections.

\section{Conclusions}

In summary, our results indicate that prophages are present in the genomes of clinical mycobacteria. Prophages are more likely to be present in mycobacteria with a rapid growth rate compared to slowly growing species. The mechanism and selective advantage of this enrichment by growth rate remains unclear. Prophages within mycobacteria do not share a core genome and are genetically distinct. Comparisons to other mycobacteriophages from PhagesDB revealed some similarities, including shared members of lettered clusters, however the largest group of integrated prophages were not assigned to a previously defined cluster. In addition, bacterial virulence genes were enriched in predicted prophages from clinical genomes relative to environmental mycobacteriophages from PhagesDB. Our comprehensive analysis of prophage frequency and their genetic composition provides insight into the capability of mycobacteriophages to transduce bacterial genes relevant to bacterial virulence, potentially influencing the progression of disease.

\section{Supplementary information}

Supplementary information accompanies this paper at https://doi.org/10. 1186/s12985-020-01394-y.

\begin{abstract}
Additional file 1: Table S1. Abundance of shell genes in prophages calculated from pangenome analysis using Roary. Shell genes are defined as genes present in 15 to $95 \%$ of genomes in a cluster. This analysis of prophages assigned to lettered clusters was only applied to clusters with 5 or more prophages.
\end{abstract}

Additional file 2. This file contains the RefSeq assembly accession, NCBI Assembly ID, Bioproject, species, and source of the complete genomes used in this study.

Additional file 3. This file contains 6 data tables used in the study. The Predicted_Prophages tab provides details about the predicted prophages including the genomic coordinates, cluster assignment, and the presence/absence of a bacterial virulence gene. The

Predicted_Prophages_Edge_Cases lists the coordinates of prophages near the ends of contigs. The Bacterial_Genes tab represents the annotated output of bacterial genes (excluding bacterial virulence genes) The Virulence_Genes represents the annotated output of bacterial virulence genes. The Cluster_Virulence tab groups the predicted prophages by cluster and virulence status. The Virulence_Gene_Origins tab represents a count of the species origin of a virulence gene in the predicted prophages.

Additional file 4: Figure S1. Relative locations of predicted prophages in M. absessus subsp. absessus and M. avium. Prophages are colored by the host origin.

Additional file 5: Figure S2. Genome wide phylogeny of $80 \mathrm{M}$. abscessus subsp. abscessus and M. abscessus subsp. boletti genomes and 5 control genomes. The heatmap on the right shows the presence (black) or absence (white) of the lettered PhagesDB clusters (x-axis). The presence of prophage in a sample is noted by a shaded box in any column except NA/Control. NA/Control shading signifies genomes without a prophage and added controls not analyzed for prophages in this study. No shading means the sample does not have a prophage in that lettered cluster.

Additional file 6: Figure S3. Phylogeny of host M. absessus subsp. massiliense genomes. The shaded boxes are located along an $x$ axis, which lists the lettered PhagesDB clusters. The presence of prophage in a sample is noted by a shaded box in any column except NA/Control. No 
shading means the sample does not have a prophage in that lettered cluster. Statistical significance was not achieved using PERMANOVA on this tree $(F=1.398, p=0.225)$.

Additional file 7: Figure S4. Phylogeny of host M. chimaera and M. intracellulare genomes. The shaded boxes are located along an $x$ axis, which lists the lettered PhagesDB clusters. The presence of prophage in a sample is noted by a shaded box in any column except NA/Control. No shading means the sample does not have a prophage in that lettered cluster. Statistical significance was not achieved using PERMANOVA on this tree $(F=1.12, p=0.321)$

\section{Abbreviations}

NTM: Nontuberculous mycobacteria; RGM: Rapidly growing mycobacteria; SGM: Slowly growing mycobacteria; MAC: M. avium complex; PATRICVF: Pathosystems Resource Integration Center virulence factor database; VFDB: Virulence Factor Database; CRISPR: Clustered regularly interspaced short palindromic repeats; ANI: Average nucleotide identity;

RAxML: Randomized Axelerated Maximum Likelihood-Next Generation; DCT: Distributed conjugal transfer

\section{Acknowledgements}

The authors would like to thank the Colorado Cystic Fibrosis Research Development Program for making the clinical NTM genomes publicly available.

\section{Authors' contributions}

CG and MS conceived the study. CG performed data analysis. SK and RD performed genome assembly. NAH generated mycobacteria phylogeny. LEE generated sequence data. CG and MS wrote the manuscript. All authors read, revised, and approved the final draft.

\section{Funding}

CG is supported by NLM 5 T15 LM009451-12. The authors would like to thank the Cystic Fibrosis Foundation for funding. MS and CG would also like to thank the Colorado Advanced Industries Grant Program for funding.

\section{Availability of data and materials}

All scripts used to derive the figures and additional preprocessing including tool overlap identification are available on the Strong Lab GitHub at https:// github.com/Strong-Lab/Prophage_In_NTM. The fasta sequences of predicted prophages are available on the Github at https://github.com/Strong-Lab/ Prophage_in_NTM/tree/master/data/fasta. The raw data of the clinical NTM draft genomes are available at BioProject 319839 and the sources of the complete genomes are listed in Supplemental File 1. Additional data tables are included in Supplementary File 2.

\section{Ethics approval and consent to participate}

$$
\text { Not applicable. }
$$

\section{Consent for publication}

Not applicable.

\section{Competing interests}

The authors declare no competing interests financial or otherwise related to this project.

Received: 14 May 2020 Accepted: 3 August 2020

Published online: 17 August 2020

\section{References}

1. Jhun BW, Moon SM, Jeon K, et al. Prognostic factors associated with longterm mortality in 1445 patients with nontuberculous mycobacterial pulmonary disease: a 15-year follow-up study. Eur Respir J. 2020;55(1): 1900798. Published 2020 Jan 2. https://doi.org/10.1183/13993003.007982019.

2. Honda JR, NA Hasan. Environmental Nontuberculous Mycobacteria in the Hawaiian Islands. 2014 [cited 2014]; Available from: http://journals.plos.org/ plosntds/article?id=10.1371/journal.pntd.0005068.

3. Covert TC, Rodgers MR, Reyes AL, Stelma GN. Occurrence of nontuberculous mycobacteria in environmental samples. Appl Environ
Microbiol. 1999;65(6):2492-6 [cited 1999 Jun 1]. Available from: http://www. ncbi.nlm.nih.gov/pubmed/10347032.

4. Gebert MJ, Delgado-Baquerizo M, Oliverio AM, Webster TM, Nichols LM, Honda JR, et al. Ecological Analyses of Mycobacteria in Showerhead Biofilms and Their Relevance to Human Health. mBio. 2018;9(5) [cited 2018 Oct 30]. Available from: http://www.ncbi.nlm.nih.gov/pubmed/30377276.

5. Honda JR, Alper S, Bai X, Chan ED. Acquired and genetic host susceptibility factors and microbial pathogenic factors that predispose to nontuberculous mycobacterial infections. Curr Opin Immunol. 2018;54:66-73 [cited 2018 Oct 21]. Available from: http://www.ncbinlm.nih.gov/pubmed/29936307.

6. Spaulding AB, Lai YL, Zelazny AM, Olivier KN, Kadri SS, Prevots DR, et al. Geographic Distribution of Nontuberculous Mycobacterial Species Identified among Clinical Isolates in the United States, 2009-2013. Ann Am Thorac Soc. 2017;14(11):1655-61 [cited 2017 Nov 1] Available from: http://www. ncbi.nlm.nih.gov/pubmed/28817307.

7. Lipner EM, Garcia BJ, Strong M. Network Analysis of Human Genes Influencing Susceptibility to Mycobacterial Infections. PloS one. 2016;11(1): e0146585 [cited 2016 Jan 11]. Available from: http://www.ncbi.nlm.nih.gov/ pubmed/26751573.

8. Runyon EH. Anonymous mycobacteria in pulmonary disease. Med Clin North Am. 1959:43(1):273-90 [cited 1959 Jan 1] Available from: http://www. ncbi.nlm.nih.gov/pubmed/13612432.

9. Slowly Growing Nontuberculous Mycobacteria (NTM) - Infectious Disease Advisor. 2020 [cited 2020 Apr 27]. Available from: https://www. infectiousdiseaseadvisor.com/home/decision-support-in-medicine/ infectious-diseases/slowly-growing-nontuberculous-mycobacteria-ntm/.

10. Penadés JR, Chen J, Quiles-Puchalt N, Carpena N, Novick RP. Bacteriophagemediated spread of bacterial virulence genes. Curr Opin Microbiol. 2015;23: 171-8 [cited 2015 Feb 19] Available from: http://www.ncbi.nlm.nih.gov/ pubmed/25528295.

11. Ravin VK, Shulga MG. Evidence for extrachromosomal location of prophage N15. Virology. 1970;40(4):800-7 [cited 1970 Apr 1]; Available from: http:// www.ncbinlm.nih.gov/pubmed/4914644.

12. Olson ME, Horswill AR. Bacteriophage Transduction in Staphylococcus epidermidis. Methods Mol Biol. 2010;1106:167-72. [cited 1970 Apr 1]; Available from: http://www.ncbi.nlm.nih.gov/pubmed/24222465.

13. Streisinger G, Emrich J, Stahl MM. Chromosome structure in phage t4, iii. Terminal redundancy and length determination. Proc Natl Acad Sci U S A. 1967;57(2):292-5. https://doi.org/10.1073/pnas.57.2.292.

14. Jiang SC, Paul JH. Gene transfer by transduction in the marine environment. Appl Environ Microbiol. 1998;64(8):2780-7 cited 1998 Aug 1]. Available from: http://www.ncbi.nlm.nih.gov/pubmed/9687430.

15. Casadevall A, Pirofski LA. Host-pathogen interactions: redefining the basic concepts of virulence and pathogenicity. Infect Immun. 1999;67(8):3703-13 [cited 1999 Aug 1]. Available from: http://www.ncbi.nlm.nih.gov/pubmed/10417127.

16. Wagner PL, Waldor MK. Bacteriophage Control of Bacterial Virulence. Infect Immun. 2002;70(8) [cited 2002 Aug 1]. Available from. https://doi.org/10. 1128/IAl.70.8.3985-3993.2002

17. León M, Bastías R. Virulence reduction in bacteriophage resistant bacteria. Front Microbiol. 2015;6:343 [cited 2015 Apr 23]. Available from: http://www. ncbi.nlm.nih.gov/pubmed/25954266.

18. Dedrick RM, Jacobs-Sera D, Bustamante CA, et al. Prophage-mediated defence against viral attack and viral counter-defence. Nat Microbiol. 2017;2 16251. Published 2017 Jan 9. https://doi.org/10.1038/nmicrobiol.2016.251.

19. Brüssow H, Canchaya C, Hardt W-D. Phages and the evolution of bacterial pathogens: from genomic rearrangements to lysogenic conversion. Microbiol Mol Biol Rev MMBR. 2004;68(3):560-602 table of contents. [cited 2004 Sep 1]. Available from: http://www.ncbi.nlm.nih. gov/pubmed/15353570.

20. Hargreaves KR, Kropinski AM, Clokie MR. What does the talking?: quorum sensing signalling genes discovered in a bacteriophage genome. PLoS One. 2014;9(1):e85131. Published 2014 Jan 24. https://doi.org/10.1371/journal. pone.0085131.

21. Williams KP. Integration sites for genetic elements in prokaryotic tRNA and tmRNA genes: sublocation preference of integrase subfamilies. Nucleic Acids Res. 2002;30(4) [cited 2002 Feb 15]. Available from. https://doi.org/10. 1093/nar/30.4.866

22. Kim Al, Ghosh P, Aaron MA, Bibb LA, Jain S, Hatfull GF. Mycobacteriophage Bxb1 integrates into the Mycobacterium smegmatis groEL1 gene. Mol Microbiol. 2003;50(2):463-73 [cited 2003 Oct 1]. Available from: http://www. ncbi.nlm.nih.gov/pubmed/14617171. 
23. Zeng H, Zhang J, Li C, et al. The driving force of prophages and CRISPR-Cas system in the evolution of Cronobacter sakazakii [published correction appears in Sci Rep. 2017 Apr 26;7:46783]. Sci Rep. 2017;7:40206. Published 2017 Jan 6. https://doi.org/10.1038/srep40206.

24. Barrangou R, Fremaux C, Deveau H, Richards M, Boyaval P, Moineau S, et al. CRISPR provides acquired resistance against viruses in prokaryotes. Sci. 2007; 315(5819):1709-12 [cited 2007 Mar 23]. Available from: http://www.ncbi.nlm. nih.gov/pubmed/17379808.

25. Russell DA, Hatfull GF. PhagesDB: the actinobacteriophage database. Bioinforma. 2017;33(5):784-6 [cited 2017 Mar 1] Available from: http://www. ncbi.nlm.nih.gov/pubmed/28365761.

26. Hatfull GF, Cresawn SG, Hendrix RW. Comparative genomics of the mycobacteriophages: insights into bacteriophage evolution. Res Microbiol. 2008;159(5):332-9. https://doi.org/10.1016/j.resmic.2008.04.008.

27. Rybniker J, Kramme S, Small PL. Host range of 14 mycobacteriophages in Mycobacterium ulcerans and seven other mycobacteria including Mycobacterium tuberculosis--application for identification and susceptibility testing. J Med Microbiol. 2006;55(Pt 1):37-42 [cited 2006 Jan 1] Available from: http://www.ncbi.n/m.nih.gov/pubmed/16388028.

28. Jacobs-Sera D, Marinelli LJ, Bowman C, Broussard GW, Guerrero Bustamante C, Boyle MM, et al. On the nature of mycobacteriophage diversity and host preference. Virology. 2012;434(2):187-201 [cited 2012 Dec 20]. Available from: http://www.ncbi.n/m.nih.gov/pubmed/23084079.

29. Seddon P, Fidler K, Raman S, Wyatt H, Ruiz G, Elston C, et al. Prevalence of Nontuberculous Mycobacteria in Cystic Fibrosis Clinics, United Kingdom, 2009. Emerg Infect Dis. 2013;19(7) [cited 2013 Jul 1] Available from: http:// wwwnc.cdc.gov/eid/article/19/7/12-0615_article.htm.

30. Zhao X, Epperson LE, Hasan NA, Honda JR, Chan ED, Strong M, et al. Complete Genome Sequence of subsp. Strain H87 Isolated from an Indoor Water Sample. Genome Announc. 2017;5(16) [cited 2017 Apr 20]. Available from: http://www.ncbi.n/m.nih.gov/pubmed/28428297.

31. Hasan NA, Davidson RM, Epperson LE, Kammlade SM, Rodger RR, Levin AR, et al. Population Genomics of Nontuberculous Mycobacteria Recovered from United States Cystic Fibrosis Patients. bioRxiv. 2019; [cited 2019 Jan 1]. Available from: https://www.biorxiv.org/content/10.1101/663559v1.

32. Käser M, Ruf MT, Hauser J, Marsollier L, Pluschke G. Optimized method for preparation of DNA from pathogenic and environmental mycobacteria. Appl Environ Microbiol. 2009;75(2):414-8. https://doi.org/10.1128/AEM.01358-08.

33. Wick RR, Judd LM, Gorrie CL, Holt KE. Unicycler: Resolving bacterial genome assemblies from short and long sequencing reads. PLoS Comput Biol. 2017; 13(6):e1005595 [cited 2017 Jun 8] Available from: http://www.ncbi.nlm.nih. gov/pubmed/28594827.

34. Davidson RM, Hasan NA, de Moura VCN, Duarte RS, Jackson M, Strong M. Phylogenomics of Brazilian epidemic isolates of Mycobacterium abscessus subsp. bolletii reveals relationships of global outbreak strains. Infect Genet Evol J Mol Epidemiol Evol Genet Infect Dis. 2013;20:292-7 [cited 2013 Dec 18]. Available from: http://www.ncbi.nlm.nih.gov/pubmed/24055961.

35. Datta G, Nieto LM, Davidson RM, Mehaffy C, Pederson C, Dobos KM, et al. Longitudinal whole genome analysis of pre and post drug treatment Mycobacterium tuberculosis isolates reveals progressive steps to drug resistance. Tuberc. 2016;98:50-5 [cited 2016 May 26]. Available from: http:// www.ncbi.nlm.nih.gov/pubmed/27156618.

36. Langmead B, Salzberg SL. Fast gapped-read alignment with Bowtie 2. Nat methods. 2012;9(4):357-9 [cited 2012 Mar 4]. Available from: http://www. ncbi.nlm.nih.gov/pubmed/22388286.

37. Li H, Handsaker B, Wysoker A, Fennell T, Ruan J, Homer N, et al. The Sequence Alignment/Map format and SAMtools. Bioinforma. 2009;25(16): 2078-9 [cited 2009 Aug 15]. Available from: http://www.ncbi.nlm.nih.gov/ pubmed/19505943.

38. Kozlov A, Darriba D, Flouri T, Morel B, Stamatakis A. RAxML-NG: A fast, scalable, and user-friendly tool for maximum likelihood phylogenetic inference. bioRxiv. 2018; [cited 2018 Jan 1]; Available from: https://www. biorxiv.org/content/early/2018/10/18/447110.

39. Yu G, Smith DK, Zhu H, Guan Y, Lam TT-Y. ggtree: anrpackage for visualization and annotation of phylogenetic trees with their covariates and other associated data. McInerny G, editor. Methods Ecol Evol. 2017;8(1). [cited 2017 Jan 1]. Available from: doi: https://doi.org/10.1111/2041-210X.12628.

40. Huerta-Cepas J, Dopazo J. Gabaldón T. ETE: a python Environment for Tree Exploration. BMC Bioinforma. 2010;11:24 [cited 2010 Jan 13] Available from: http://www.ncbi.nlm.nih.gov/pubmed/20070885.
41. Laslett D, Canback B. Aragorn, a program to detect tRNA genes and tmRNA genes in nucleotide sequences. Nucleic acids Res. 2004;32(1):11-6 [cited 2004 Jan 2] Available from: http://www.ncbi.nlm.nih.gov/pubmed/14704338.

42. Bland C, Ramsey TL, Sabree F, Lowe M, Brown K, Kyrpides NC, et al. CRISPR recognition tool (CRT): a tool for automatic detection of clustered regularly interspaced palindromic repeats. BMC Bioinforma. 2005;8:209 [cited 2005] Available from: http://www.ncbi.nlm.nih.gov/pubmed/17577412http://www. pubmedcentral.nih.gov/articlerender.fcgi?artid=1924867\&tool= pmcentrez\&rendertype=abstract.

43. Arndt D, Grant JR, Marcu A, Sajed T, Pon A, Liang Y, et al. PHASTER: a better, faster version of the PHAST phage search tool. Nucleic acids Res. 2016; 44(W1):W16-21 [cited 2016 Jul 8] Available from: http://www.ncbi.nlm.nih. gov/pubmed/27141966.

44. Sullivan MB, Hurwitz BL, Roux S, Enault F. VirSorter: mining viral signal from microbial genomic data. PeerJ. 2015;3 [cited 2015 May 28]. Available from: https://peerj.com/articles/985/.

45. Fan X, Xie L, Li W, Xie J. Prophage-like elements present in Mycobacterium genomes. BMC Genomic. 2014;15:243 [cited 2014 Mar 27] Available from: http://www.ncbi.nlm.nih.gov/pubmed/24673856.

46. Rognes T, Flouri T, Nichols B, Quince C, Mahé F. VSEARCH: a versatile open source tool for metagenomics. PeerJ. 2016;4:e2584 [cited 2016 Oct 18] Available from: http://www.ncbi.nlm.nih.gov/pubmed/27781170.

47. Kloepper $\mathrm{TH}, \mathrm{Huson} \mathrm{DH}$. Drawing explicit phylogenetic networks and their integration into SplitsTree. BMC Evol Biol. 2008;8:22 [cited 2008 Jan 24]. Available from: http://www.ncbi.nlm.nih.gov/pubmed/18218099.

48. Delcher AL, Salzberg SL, Phillippy AM, et al. Curr Protoc Bioinforma. 2003; chapter 10:unit 10.3.[cited 2003 Feb 1] Available from: http://www.ncbi.nlm. nih.gov/pubmed/18428693.

49. Hatfull GF, Jacobs-Sera D, Lawrence JG, et al. Comparative genomic analysis of 60 Mycobacteriophage genomes: genome clustering, gene acquisition, and gene size. J Mol Biol. 2010;397(1):119-43. https://doi.org/10.1016/j.jmb. 2010.01.011.

50. Buchfink B, Xie C, Huson DH. Fast and sensitive protein alignment using DIAMOND. Nat methods. 2015;12(1):59-60 [cited 2015 Jan 17]. Available from: http://www.ncbi.nlm.nih.gov/pubmed/25402007.

51. Hyatt D, Chen G-L, Locascio PF, Land ML, Larimer FW, Hauser LJ. Prodigal: prokaryotic gene recognition and translation initiation site identification. BMC Bioinforma. 2010;11:119 [cited 2010 Mar 8]. Available from: http://www. ncbi.nlm.nih.gov/pubmed/20211023.

52. Mauri M, Elli T, Caviglia G, Uboldi G, Azzi M. RAWGraphs: A Visualisation Platform to Create Open Outputs. In: Proceedings of the 12th Biannual Conference on Italian SIGCHI Chapter - CHItaly '17: ACM Press; 2015. p. 1-5. [cited 2015] Available from: http://dl.acm.org/citation.cfm?doid=31255 71.3125585 .

53. Seemann T. Prokka: rapid prokaryotic genome annotation. Bioinforma. 2014; 30(14):2068-9 [cited 2014 Jul 15]. Available from: http://www.ncbi.nlm.nih. gov/pubmed/24642063.

54. Chen L. VFDB: a reference database for bacterial virulence factors. Nucleic Acids Res. 2004;33(Database issue) [cited 2004 Dec 17] Available from: https://academic.oup.com/nar/article-lookup/doi/10.1093/nar/gki008.

55. Wattam AR, Abraham D, Dalay O, Disz TL, Driscoll T, Gabbard JL, et al. PATR IC, the bacterial bioinformatics database and analysis resource. Nucleic acids Res. 2014;42(Database issue):D581-91 [cited 2014 Jan 12]. Available from: http://www.ncbi.nlm.nih.gov/pubmed/24225323.

56. Sonnhammer EL, Eddy SR, Birney E, Bateman A, Durbin R. Pfam: multiple sequence alignments and HMM-profiles of protein domains. Nucleic acids Res. 1998;26(1):320-2 [cited 1998 Jan 1]. Available from: http://www.ncbi. nlm.nih.gov/pubmed/9399864.

57. McKinney W. Data Structures for Statistical Computing in Python. Proc 9th Python Sci Conf. 2006;1697900:51-6.

58. Hunter JD. "Matplotlib: A 2D Graphics Environment," in Computing in Science \& Engineering. 2007;9(3):90-5. https://doi.org/10.1109/MCSE.2007.55.

59. Page AJ, Cummins CA, Hunt M, Wong VK, Reuter S, Holden MTG, et al. Roary: rapid large-scale prokaryote pan genome analysis. Bioinforma. 2015; 31(22):3691-3 [cited 2015 Nov 15]. Available from: http://www.ncbi.nlm.nih. gov/pubmed/26198102.

60. Seni G, Elder JF. Ensemble Methods in Data Mining: Improving Accuracy Through Combining Predictions. Synth Lect Data Min Knowl Discov. 2010; 2(1) [cited 2010 Jan 1] Available from: http://www.morganclaypool.com/doi/ abs/10.2200/S00240ED1V01Y200912DMK002. 
61. He L, Fan X, Xie J. Comparative genomic structures of Mycobacterium CRIS PR-Cas. J Cell Biochem. 2012;113(7):2464-73 [cited 2012 Jul 1] Available from: http://www.ncbi.nlm.nih.gov/pubmed/22396173.

62. Nabergoj D, Modic P, Podgornik A. Effect of bacterial growth rate on bacteriophage population growth rate. MicrobiologyOpen. 2018;7(2):e00558 [cited 2018 Apr 1] Available from: http://www.ncbi.nlm.nih.gov/pubmed/291 95013.

63. Wiggins BA, Alexander M. Minimum bacterial density for bacteriophage replication: implications for significance of bacteriophages in natural ecosystems. Appl Environ Microbiol. 1985;49(1):19-23. https://doi.org/10. 1128/AEM.49.1.19-23.1985.

64. Pedulla ML, Ford ME, Houtz JM, Karthikeyan T, Wadsworth C, Lewis JA, et al. Origins of highly mosaic mycobacteriophage genomes. Cell. 2003;113(2): 171-82 [cited 2003 Apr 18]. Available from: http://www.ncbi.nlm.nih.gov/ pubmed/12705866.

65. González-Pérez M, Mariño-Ramírez L, Parra-López CA, Murcia MI, Marquina B, Mata-Espinoza D, et al. Virulence and immune response induced by Mycobacterium avium complex strains in a model of progressive pulmonary tuberculosis and subcutaneous infection in BALB/c mice. Infect Immun. 2013;81 (11):4001-12 [cited 2013 Nov 19]. Available from: http://www.ncbi. nlm.nih.gov/pubmed/23959717.

66. Sapriel G, Konjek J, Orgeur M, Bouri L, Frézal L, Roux A-L, et al. Genomewide mosaicism within Mycobacterium abscessus: evolutionary and epidemiological implications. BMC Genomic. 2016;17:118 [cited 2016 Feb 17]. Available from: http://www.ncbi.nlm.nih.gov/pubmed/26884275.

67. Malachowa N, FR DL. Mobile genetic elements of Staphylococcus aureus. Cell Mol life Sci CMLS. 2010;67(18):3057-71 [cited 2010 Sep 29] Available from: http://www.ncbi.nlm.nih.gov/pubmed/20668911.

\section{Publisher's Note}

Springer Nature remains neutral with regard to jurisdictional claims in published maps and institutional affiliations.

Ready to submit your research? Choose BMC and benefit from:

- fast, convenient online submission

- thorough peer review by experienced researchers in your field

- rapid publication on acceptance

- support for research data, including large and complex data types

- gold Open Access which fosters wider collaboration and increased citations

- maximum visibility for your research: over $100 \mathrm{M}$ website views per year

At $\mathrm{BMC}$, research is always in progress.

Learn more biomedcentral.com/submissions 